dencia para recomendar el rastreo selectivo a niños con alto riesgo de intoxicación (niños que viven o visitan periódicamente viviendas construidas con anterioridad a 1950 con deterioro de su pintura, o en las cuales se planea o realizó recientemente alguna refacción o remodelación; en aquellos con hermanos, convivientes o afines con intoxicación por plomo; conviviente con un adulto cuya ocupación o hobbie implique exposición sustancias con plomo; o residentes en las cercanías de industrias o autopistas con alto tránsito) (B); y evidencia insuficiente para recomendar a favor o en contra del rastreo en la población general (C).

articulo.php?cod_producto=956

2. Ciapponi A. Nueva guía de cuidados preventivos de la Fuerza de Tareas de Estados Unidos (primera entrega). Evid actual pract ambul 2005;8:179-182. Disponible en: http://www.foroaps.org/files/nueva\%20fuerza.pdf

3. Adaptado por Ciapponi A de la. Nueva guía de cuidados preventivos de la Fuerza de Tareas de Estados Unidos (segunda entrega). Evid. actual. práct. ambul; 9(1): 26-30, Ene-Feb 2006. Disponible en: http://www.foroaps.org/files/cuida\%20preven\%202\%20parte.pdf

4. Centers for disease control and prevention. Tuberculosis. Testing \& dianosis (en línea). Ultima actualización 25/06/2010. Atlanta GA, EE.UU. Disponible en http://www.cdc.gov/tb/topic/testing/default.htm

\title{
Noticiero Epidemiológico: \\ Se retira del mercado medicamentos que contengan Sibutramina
}

\begin{abstract}
Débora J. Szeinman
La sibutramina, un fármaco inhibidor de la recaptación de serotonina y noradrenalina, fue aprobada en noviembre del 2007 para la pérdida de peso y el mantenimiento del mismo en personas obesas y en aquellas con sobrepeso y factores de riesgo para enfermedad cardiaca. Esta aprobación se baso en datos clínicos que mostraban que las personas que utilizaban sibutramina perdían un $5 \%$ más de peso que aquellas que usaban placebo y basaban su tratamiento en dieta y actividad física solamente.
\end{abstract}

Como consecuencia de los reportes recibidos de eventos cardiovasculares, fundamentalmente aumento de la presión sanguínea y de la frecuencia cardiaca, la sibutramina fue objeto de revisión por parte de distintas agencias regulatorias de medicamentos a nivel mundial ${ }^{1}$. Los resultados del estudio de seguridad SCOUT (Sibutramine Cardiovascular OUTcome trial), rea-lizado en una población de pacientes con sobrepeso $u$ obesidad y con antecedentes de enfermedad cardiovas- cular preexistente y/o diabetes mellitus tipo II demostraron, en el grupo que recibió sibutramina (en comparación al que recibió placebo), un aumento del $16 \%$ del riesgo de eventos serios cardiovasculares, incluyendo: infarto agudo de miocardio no fatal, accidente cerebrovascular no fatal, reanimación después de episodios de paro cardiaco, y muerte.

En base a estos resultados la Administración Nacional de Medicamentos, Alimentos y Tecnología Médica (ANMAT) ${ }^{2}$ y la administración Estadounidense de medicamentos y alimentos (sigla en inglés, FDA) ${ }^{3}$ han suspendido la comercialización y el uso de todas las especialidades medicinales que contengan en su formulación la droga sibutramina.

Por todo lo expuesto tanto la ANMAT como la FDA anuncian en Octubre de 2010 el retiro de la droga del mercado y recomiendan a los médicos dejar de prescribir sibutramina y a los pacientes dejar de tomar este medicamento.

Referencias

1. Villalón G. Noticiero epidemiológico: Advertencia sobre isotretinoina y sobre sibutramina. Evid. actual. práct. ambul; 13(1):10 ene-mar 2009.

2. Administración Nacional de Medicamentos, Alimentos y Tecnología. Comunicado de prensa. Suspensión de Sibutramina, 19 de octubre, 2010 (en línea) Disponible en URL: http://www.anmat.gov.ar/Comunicados/Sibutramina suspension profesionales. pdf (ultimo acceso 08/11/2010).

3. U.S. Food and Drug Administration. FDA Drug Safety Communication: FDA Recommends Against the Continued Use of Meridia (sibutramine) (en línea) Disponible en URL: http://www.fda.gov/Drugs/DrugSafety/ucm228746.htm\#Safety_Announcement 